Canadian

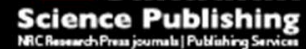

Canadian Journal of Microbiology Revue canadienne de de microbiologie

The Essential Activities of the Bacterial Sigma Factor

\begin{tabular}{|r|l|}
\hline Journal: & Canadian Journal of Microbiology \\
\hline Manuscript ID & cjm-2016-0576 \\
\hline Manuscript Type: & Review \\
\hline Complete List of Authors: & $\begin{array}{l}\text { Davis, Maria C.; University of New Brunswick, Department of Biology } \\
\text { Kesthely, Christopher A.; University of New Brunswick, Department of } \\
\text { Biology } \\
\text { Franklin, Emily A.; University of New Brunswick, Department of Biology } \\
\text { MacLellan, Shawn R.; University of New Brunswick, Department of Biology }\end{array}$ \\
\hline Keyword: & transcription, bacteria, RNA polymerase, sigma factor, gene expression \\
\hline
\end{tabular}

SCHOLARONE ${ }^{m}$

Manuscripts 


\section{The Essential Activities of the Bacterial Sigma Factor}

Maria C. Davis, Christopher A. Kesthely, Emily A. Franklin, and Shawn R. MacLellan\#.

Affiliations:

Department of Biology

University of New Brunswick

Fredericton, New Brunswick, Canada

\#Corresponding Author:

Shawn R. MacLellan

srm@unb.ca 


\section{Abstract}

Transcription is the first and most heavily regulated step in gene expression. Sigma $(\sigma)$ factors are general transcription factors that reversibly bind RNA polymerase and mediate transcription of all genes in bacteria. $\sigma$ factors play three major roles in the RNA synthesis initiation process, they: a) target RNAP polymerase holoenzyme to specific promoters, b) melt a region of doublestranded promoter DNA and stabilize it as a single-stranded open complex, and c) interact with other DNA-binding transcription factors to contribute complexity to gene expression regulation schemes. Recent structural studies have demonstrated that when $\sigma$ factors bind promoter DNA, they capture one or more nucleotides that are flipped out of the helical DNA stack and this stabilizes the promoter open-complex intermediate that is required for the initiation of RNA synthesis.

This review describes the structure and function of the $\sigma^{70}$ family of $\sigma$ proteins and the essential roles they play in the transcription process.

Key words: transcription, bacteria, RNA polymerase, sigma factor, gene expression 


\section{Introduction}

In all cells, a DNA-dependent enzyme complex called RNA polymerase (RNAP) carries out RNA synthesis (transcription). The bacterial RNAP holoenzyme nominally consists of 6 subunits: the $\beta$ and $\beta^{\prime}$ subunits that form the active site, an $\alpha$ subunit dimer, an $\omega$ subunit, and a reversiblybinding subunit called $\sigma$ (Fig. 1a) (Feklístov et al. 2014, Murakami 2015, Ruff et al. 2015). In most bacterial species, several different $\sigma$ factors recognize cognate promoter sequences and ensure that all functional genes in the genome are expressed when required for cellular viability. This review delineates three conceptually distinct activities of the largest, most ubiquitous family of $\sigma$ factors, the $\sigma^{70}$ family.

\section{The transcription process}

The transcription process (Fig. 2) begins with the assembly of polymerase subunits $\left(\alpha_{2} \beta \beta^{\prime} \omega\right)$ into a core RNAP complex. Binding of a $\sigma$ factor subunit completes the holoenzyme and targets the complex to cognate promoter sequences upstream of genes that comprise the regulon of the $\sigma$ factor (Murakami and Darst 2003, Feklístov et al. 2014). In addition to acting as a specificity factor, the $\sigma$ factor plays a crucial role in melting the -10 region of the doublestranded promoter DNA, and binding the non-template strand DNA. This traps the region in a single-stranded bubble called an open-complex (Juang and Helmann 1994, DeHaseth and Helmann 1995, Panaghie et al. 2000) (Fig. 2, step 4). The template strand of the -10 element enters the active site of the polymerase (formed at the intersection between the large $\beta$ and $\beta^{\prime}$ subunits) and primer-independent RNA synthesis initiates. Often after several abortive initiation events that produce RNA transcripts 2 to 15 nucleotides in length (Gralla et al. 1980, 
Goldman et al. 2009), RNAP holoenzyme escapes the promoter, $\sigma$ dissociates, and the elongation phase of transcription ensues (Fig. 2, step 6). Downstream of an open reading frame, termination of transcription is accomplished by the formation of an RNA stem-loop structure or through the action of a protein called Rho (Gusarov and Nudler 1999, Ciampi 2006).

The polymerization of ribonucleotides is solely the activity of core RNAP and the role of the $\sigma$ factor as a targeting or specificity factor is well-recognized amongst microbiologists. In this review, we highlight two additional $\sigma$ factor activities: 1) their interaction with other transcription factors and the influence of these interactions on regulated gene expression, and 2) the role of $\sigma$ in melting duplex promoter DNA. We especially focus on this latter role because core RNAP is unable to initiate transcription from double-stranded DNA. Thus, in addition to the essential function of targeting RNAP holoenzyme to the gene promoter, $\sigma$ factors are also mechanistically essential for initiating transcription.

\section{Overview of sigma factor types}

$\sigma$ factors fall into two structurally and evolutionarily-distinct families: the $\sigma^{54}$ (sometimes called RpoN) family (Zhang and Buck 2015) and the $\sigma^{70}$ family (variously called RpoD or $\sigma^{A}$ but named after the canonical Escherichia coli $70 \mathrm{kDa}$ vegetative $\sigma$ factor) (Paget and Helmann 2003, Feklístov et al. 2014). Many, but not all, bacterial genomes carry one or two $\sigma^{54}$ genes and these $\sigma$ factors are frequently involved in regulating the expression of genes involved in nitrogen metabolism. By contrast, all bacteria express at least one $\sigma^{70}$-family protein called the primary, housekeeping, or vegetative $\sigma$ factor that is responsible for the expression of most or all unconditionally essential genes. Free-living bacterial species frequently synthesize multiple 
distinct $\sigma^{70}$-family $\sigma$ factors including the essential primary (or Group I) $\sigma$ factor and a variable number of alternative $\sigma$ factors that can be categorized as Group II, III, or IV proteins (Lonetto et al. 1992, Missiakas and Raina 1998, Gruber and Gross 2003). In most bacteria, Group II and III $\sigma$ factors collectively regulate the expression of a large number of genes including those involved in general stress responses, flagellar structure and function, chemotaxis, and developmental processes such as endospore formation. Group IV (or Extracytoplasmic Function, ECF) proteins are numerically the largest group of $\sigma^{70}$-family $\sigma$ factors and regulate the expression of genes that ensure the cell can mount an appropriate physiological response to varying and often stressful environmental conditions. All functional gene promoters in a genome are recognized by one (sometimes more than one) $\sigma$ factor. Thus, $\sigma$ factors are the master regulators of all gene expression in bacteria and collectively the cellular complement of $\sigma$ factors help initiate transcription from every gene.

\section{The structure of $\sigma^{70}$ family proteins}

Beginning with the first detailed X-ray crystallographic structure reported for a portion of $E$. coli $\sigma^{70}$ in 1996 (Malhotra et al. 1996) and a nearly complete imaging of Thermus aquaticus $\sigma^{A}$ in 2002 (Campbell et al. 2002), several structures have been reported for bacterial $\sigma$ factors, RNAP holoenzymes, and these proteins complexed with various promoter DNA constructs [e.g. (Bae et al. 2015, Zuo and Steitz 2015, Liu et al. 2016, Feng et al. 2016) and see (Murakami 2015) for a historical summary of solved structures]. For the Group I vegetative $\sigma^{70}$ sigma factors, frequent targets for structural analyses have included the highly conserved T. aquaticus and E. coli vegetative $\sigma$ factors. 
Group I $\sigma^{70}$ consists of four folded domains (called $\sigma_{1}, \sigma_{2}, \sigma_{3}, \sigma_{4}$ ) connected by linker sequences of varying lengths (see Fig. 1a for a schematic representation) (Malhotra et al. 1996, Campbell et al. 2002, Murakami et al. 2002, Vassylyev et al. 2002). All of these domains mediate either protein-protein interactions with core RNAP, important interactions with promoter DNA, or catalyze mechanistically important steps in the transcription initiation process. Prior to the detailed structural information emerging from crystallographic analyses, peptide segments of $\sigma$ were delineated by regions (regions 1, 2, 3, and 4) and sub-regions on the basis of functional roles and amino acid sequence conservation (Gribskov and Burgess 1986, Helmann and Chamberlin 1988) (Fig. 1b). These regional peptide designations, although in some sense superseded by more definitive tertiary structural determinations, are still useful for indicating contiguous, functionally important segments of amino acids within the folded domains. For example, amino acids within region 2.3 (a small component of $\sigma_{2}$ ) are especially important for stabilizing the promoter open-complex that is essential for the initiation of transcription (Juang and Helmann 1994, DeHaseth and Helmann 1995).

Of the four $\sigma^{70}$ domains, $\sigma_{2}$ and $\sigma_{4}$ are the most functionally important portions of the $\sigma$ factor. A segment of amino acids in domain $\sigma_{4}$ (region 4.2, Fig. 1b) forms a helix-turn-helix motif that recognizes and interacts with the -35 promoter element. This domain also forms a proteinprotein interaction with the $\beta$ subunit of core RNAP that is required for holoenzyme formation (Geszvain et al. 2004). $\sigma_{2}$ likewise makes essential contact with core via the $\beta^{\prime}$ subunit (Arthur and Burgess 1998) and participates in essential functional interactions with the -10 element (Fig. 1c). The smallest $\sigma^{70}$ proteins, the $\sim 20 \mathrm{kDa}$ Group IV or ECF $\sigma$ factors consist only of 
domains $\sigma_{2}$ and $\sigma_{4}$ (Campbell et al. 2003), highlighting the functional importance of these domains.

\section{The activities of the bacterial $\sigma$ factor}

\section{i) the $\sigma$ factor as a specificity factor}

$\sigma$ factors are general transcription factors that were first identified in 1969 as proteins that stimulate transcription by RNAP (Burgess et al. 1969). Several experimental pursuits demonstrated their importance in recognizing promoter DNA and targeting RNAP to this DNA (Dickson et al. 1975, Gardella et al. 1989, Siegele et al. 1989, Zuber et al. 1989). See Feklístov et al. (2014) and references therein, for a historical perspective on the discovery of $\sigma$ factor and its function and Hook-Barnard and Hinton (2007) for a detailed review of promoter structure and characterization. Though by no means invariant, the most common arrangement for $\sigma^{70}$ promoters is the presence of two highly conserved sequences situated -10 and -35 nucleotides upstream of the +1 transcription start site (Fig. 1c). Despite an estimated 2 billion years since the divergence of Gram negative and Gram positive cell types from a common ancestor (Doolittle et al. 1996, Feng et al. 1997), high conservation remains amongst the -10 and -35 subsequences found in bacterial promoters recognized by the primary $\sigma$ factor, as illustrated by $E$. coli (Fig. 1d) and Bacillus subtilis (Fig. 1e) -10 element sequence logos. Other promoter elements such as the UP element (Aiyar et al. 1998), the extended -10 element (Keilty and Rosenberg 1987, Barne et al. 1997, Sanderson et al. 2003), and the discriminator element (Travers 1980, Haugen et al. 2006) (Fig. 1c) are functionally important in many sequences. An overly simplified view of $\sigma$ factor specificity is that each $\sigma$ factor in a cell (these may number 
between 1 and >100 proteins depending on the species) (Feklístov et al. 2014) predominantly recognizes a unique -10 and -35 sequence combination and all of the genes preceded by this unique sequence constitute the regulon (the collection of regulated genes) specific for that $\sigma$ factor. In reality, some distinct $\sigma$ factors share very similar recognition elements with others and significant cross-recognition can occur. Achieving near-specific temporal patterns of gene expression in cells is dependent on several factors including the relative concentration of a specific free $\sigma$ factor in the cytoplasm, its relative affinity for core RNAP (these two parameters influence its ability to compete for limiting amounts of available core), and the action of accessory proteins, such as activator transcription factors.

Interestingly, study of the E. coli ECF factor $\sigma^{\mathrm{E}}$ led to the discovery that a short amino acid loop connecting two helices in $\sigma_{2}$ region 2.3 of Group IV $\sigma$ factors acts as a specificity determinant that recognizes a key single base at position -10 in the promoter -10 element. The sequence of this specificity loop is poorly conserved amongst ECF $\sigma$ factors and variant loop sequences mediate recognition of a specific -10 element carrying a specific -10 base. In this way, multiple ECF $\sigma$ factors in a cell can differentiate between very similar promoter sequences (Campagne et al. 2014).

$\sigma$ factor specifically recognizes and binds the -35 hexanucleotide sequence through multiple helix-turn-helix/major groove and non-specific interactions (Gardella et al. 1989, Siegele et al. 1989, Kenney and Moran 1991, Campbell et al. 2002, Lane and Darst 2006). It was a reasonable expectation that a similarly well-defined interaction would occur between $\sigma$ and the conserved double-stranded -10 element although biochemical analyses have strongly implied that single- 
stranded non-template DNA might be a greater determinant of $\sigma /-10$ binding (Qiu and Helmann 1999, Fenton and Gralla 2003, Mekler and Severinov 2013). As noted (Hook-Barnard and Hinton 2007), few mutations in $\sigma$ factor that suppress -10 mutations have been isolated. Recent structural analyses suggest that there are fewer intermolecular interactions between domain $\sigma_{2}$ and duplex -10 element DNA and these are primarily of a non-specific nature (Feklistov and Darst 2011). At least for primary Group $1 \sigma$ factors, significant recognition and binding of the -10 element therefore seems to be dependent on, and concomitant with, strand opening. Although the -10 and -35 sequences are the most important promoter elements for $\sigma$ factor (holoenzyme) recognition and binding, $\sigma$ and also RNAP subunits make additional contacts with promoter DNA (Ruff et al. 2015) and interaction patterns are probably dynamic throughout the transcription initiation process (Fig. 2).

\section{ii) $\sigma$ factor interaction with transcription factors}

Most, but not all, transcription activator proteins stimulate holoenzyme-dependent transcription by binding to recognition sequences in the promoter. Theoretically, they can stimulate transcription by promoting progression to any intermediate in the initiation mechanism. In practice, it appears that recruitment of RNAP to promoters is the dominant mechanism that leads to activation (Lee et al. 2012). Other established mechanisms of transcription activation include promotion of holoenzyme formation [e.g. Crl, (Banta et al. 2013)], regulation of the isomerization from closed double-stranded DNA to single-stranded open complex [e.g. $\lambda c l$, (Dove et al. 2000)], and the enhancement of polymerase promoter escape [e.g. Btr, (Gaballa et al. 2012)]. In some specific cases, certain promoters possess 
suboptimal spacing between the -35 and -10 recognition elements and binding of an activator protein bends or twists the promoter DNA to establish an optimal spacing between these elements, thus permitting holoenzyme binding (Heldwein and Brennan 2001, Brown et al. 2003). More often, activators make specific intermolecular interactions with DNA and RNAP and this stabilizes the holoenzyme-promoter interaction. In essence, most activator-dependent promoters carry sub-optimal -10 and -35 sequences and this limits transcription in the absence of activator binding (Lee et al. 2012). The existence of promoters with sub-optimal -10 or -35 sequences is of course not a defect; it renders gene expression conditional on the activator protein, itself functionally activated as a response to some physicochemical cue (e.g. small ligand binding) that constitutes a signal to which the cell must mount a transcriptional response.

Most activator protein DNA-binding sites overlap or lie upstream of the -35 promoter element. Accordingly, for those activators that at some promoters directly contact $\sigma$ factor (e.g. $\lambda c l, C r p$, AraC-family activators and others) (Niu et al. 1996, Lonetto et al. 1998, Dove et al. 2000, 2003) the interaction site is usually with domain $\sigma_{4}$, the moiety of $\sigma$ that binds to the -35 subsequence (Lee et al. 2012) (Fig. 1b, c).

Activators that appear to act proximal to the -10 element/ $\sigma_{2}$ region have also been identified. These include the essential Mycobacterial activator RbpA (Paget et al. 2001, Tabib-Salazar et al. 2013, Hubin et al. 2015), GrgA from Chlamydia trachomatis (Bao et al. 2012), E. coli Crl (Pratt and Silhavy 1998), and B. subtilis RsoA (MacLellan et al. 2008). Crl stimulates $\sigma^{\text {S }}$-dependent transcription at least in part by interacting with $\sigma^{\varsigma}$ and promoting its assembly into holoenzyme 
(Banta et al. 2013, 2014). RsoA, which possesses some sequence similarity to $\sigma$, interacts with the amino terminus of the ECF-like $\sigma$ factor SigO and displays weak interaction activity with the clamp helices region of the RNAP $\beta^{\prime}$ subunit (MacLellan et al. 2009, Xue et al. 2016). The mechanism of RsoA action is not currently known, but it is co-required along with SigO for transcription from target promoters in vivo (MacLellan et al. 2008), and for open-complex formation in vitro (MacLellan et al. 2009).

Many $\sigma$ factors (particularly Group IV proteins) are negatively regulated by membrane or cytoplasmic proteins called anti-sigma factors (Missiakas and Raina 1998, Helmann 1999, Paget 2015). In the absence of a specific environmental cue, anti- $\sigma$ factors bind and sequester $\sigma$ factors from core RNAP. With the imposition of a specific physicochemical signal, anti- $\sigma$ factor function is abrogated through targeted proteolysis (Heinrich and Wiegert 2009), by structural modification (Kang et al. 1999, Paget 2015), or by the action of an anti-anti- $\sigma$ factor (Pané-Farré et al. 2005, Francez-Charlot et al. 2009) and the $\sigma$ factor is released into the cytoplasm and available for holoenzyme formation and transcription. Either directly or indirectly, anti- $\sigma$ factors are important modules for sensory perception in bacteria. They can be thought of as non-DNA binding transcription factors that repress transcription by preventing a productive $\sigma$ factor-core RNAP interaction, rather than by directly blocking RNAP access to promoter DNA, as in the case with most DNA-binding repressors (Browning and Busby 2004). Anti- $\sigma$ factor/ $\sigma$ factor partners are critical for sensing and responding to variations in the environment. Accordingly they, along with two-component regulatory systems and independently acting activator and repressor proteins have been called the three pillars of signal transduction in bacteria (Staron et al. 2009). 
As a target for interaction with transcription factors, $\sigma$ factor becomes part of a signal transduction network involving activators, repressors, chemical secondary messengers, and other regulatory molecules. Whatever the specific consequence of $\sigma$ factor interaction with other transcription factors, these protein-protein interactions lend specificity, flexibility, and temporal complexity to regulated gene expression schemes in bacteria.

\section{iii) Promoter DNA melting and stabilization of the open-complex}

Perhaps less widely understood than the well-known role of $\sigma$ as a specificity factor that targets RNAP holoenzyme to cognate gene promoters, $\sigma$ factor also plays an essential role in the transcription initiation mechanism. After holoenzyme forms a productive interaction with promoter DNA, the double-stranded -10 region (e.g. 5'- $T_{-12} A_{-11} T_{-10} A_{-9} A_{-8} T_{-7}-3^{\prime}$, see Fig. $1 d$, e) of the promoter is situated close to the active site of the polymerase formed at the intersection between the $\beta$ and $\beta^{\prime}$ subunits. A single-stranded open complex of about 12-14 nucleotides is established extending from nucleotides -11 to +1 (or slightly downstream of the +1 transcription start) (Fig. 3a). $\sigma$ factor bound to the non-template strand traps the region in an open complex and allows the single-strand template DNA to enter the polymerase active site where ribonucleotide polymerization initiates from the +1 start site. $\sigma$ factor accomplishes this strand dissociation without the use of a hydrolysable energy source. Recent detailed crystal and solution structural analyses are shedding increasing light on this process.

Co-crystallization of the $T$. aquaticus $\sigma_{2}$ domain with a non-template -10 sequence (Feklistov and Darst 2011) showed that two bases (a thymine at position -7 and adenine at position -11 ) are captured by binding pockets in $\sigma$ (Fig. 3a, b). Thus, these two nucleotides flip out of a single- 
stranded stack and into their respective pockets. The majority of specific intermolecular interactions (Fig. 3 b) between amino acid side chains from sub-region 2.3 (see Fig. 4c) and the -10 element nucleotides occurs with these flipped out bases (Feklistov and Darst 2011). In the case of $A_{-11}$, several region 2.3 amino acids comprise a pocket that accommodates the purine base (Fig. 3a, b and $4 a, b$ ) and participate in stacking, cation- $\pi$, hydrogen bond, and Van der Waals interactions (Fig. 3b). A proper fit between the -7T and -11A bases and their respective binding pockets may in part explain the rather strict conservation of these residues in the -10 elements of most bacterial promoters, including those in E. coli (Fig. 1d) and B. subtilis (Fig. 1e) (Feklistov and Darst 2011, Zhang et al. 2012). An understanding of the importance of aromatic region 2.3 amino acids for promoter melting and nucleotide $-11 \mathrm{~A}$ for -10 recognition and nucleation of strand-opening first emerged from early studies with B. subtilis RNAP (Juang and Helmann 1994, Rong and Helmann 1994, Qiu and Helmann 1999) and E. coli RNAP (Panaghie et al. 2000). In the $A_{-11}$ binding pocket, a tyrosine (Y253, Fig. 4b) stacks against the purine base (Feklistov and Darst 2011), confirming conclusions from earlier biochemical studies using a base analog at position -11 (Lim et al. 2001, Tsujikawa et al. 2002a). Following the work of Feklistov and Darst (2011), a structure solved by Ebright and co-workers consisting of nearly full-length $T$. aquaticus $\sigma^{\mathrm{A}}$ and a more extensive open-complex construct confirmed these base-flipping events and indicated two additional flipped out bases at positions -6 and +2 (Zhang et al. 2012).

Group IV or ECF $\sigma$ factors have more variable region 2.3 sequences and their cognate promotors have -10 recognition sequences that differ from the Group $1 \sigma$ factors. Vorholt and co-workers (Campagne et al. 2014) solved the structure of the $E$. coli ECF $\sigma$ factor $\sigma^{\mathrm{E}} \sigma_{2}$ domain complexed with its cognate non-template strand $\left(5^{\prime}-\mathrm{G}_{-12} \mathrm{~T}_{-11} \mathrm{C}_{-10} \mathrm{~A}_{-9} \mathrm{~A}_{-8} \mathrm{~A}_{-7}-3^{\prime}\right)$ and showed that a 
single cytosine base (position -10) is flipped out of the single-strand stack and captured by region 2.3 amino acids.

Base-flipping (Roberts and Cheng 1998), the $\sim 180^{\circ}$ rotation of a base out of a single or doublestranded helical stack is a mechanism used by several proteins, especially DNA-repair enzymes, to expose the base to further action. In the case of $\sigma$, the further action is to capture the flipped-out base into a binding pocket (Feklistov and Darst 2011, Zhang et al. 2012, Campagne et al. 2014, Karpen and deHaseth 2015) and this probably nucleates sequential base unpairing events and in part stabilizes the open-complex intermediate. Base-flipping frequently involves intercalation or strand invasion by an aromatic amino acid side chain. At least for some enzymes, this imparts specificity to the base-flipping reaction and also stabilizes the extrahelical conformation (Hendershot and O'Brien 2014). A recent structure of T. aquaticus holoenzyme complexed with a complete transcription bubble (Bae et al. 2015) suggests a role for the region 2.3 W256 residue (see Fig. 4b). For Group $1 \sigma^{70}$ proteins, this invariant tryptophan potentially acts as a steric mimic, stacking against the -12 thymine planar base in a space vacated by the flipped out adenine at position -11 (Bae et al. 2015). Intercalation of $\sigma$ region 2.3 aromatic amino acid side chains was a mechanistic possibility first postulated nearly 30 years ago (Helmann and Chamberlin 1988).

A unifying picture of $\sigma$ factor function is emerging, given the high conservation amongst most primary $\sigma^{70}$ promoter -10 elements from a broad range of bacteria, especially the critical $T_{-7}$ and $A_{-11}$ nucleotides, and the demonstration that a single nucleotide $\left(C_{-10}\right)$ is also captured from the helical stack by a Group IV $\sigma$ factor. Although the participating $\sigma$ region 2.3 amino acids and 
target nucleotides may vary amongst $\sigma$ factor groups and their cognate promoters, the flipping out of one or more nucleotides from the -10 element duplex appears to be a general mechanism employed by $\sigma$ factors to establish that critical transcription initiation intermediate, the open-complex.

\section{Concluding Remarks}

$\sigma$ factors mediate all gene expression in bacteria. A variable assemblage of Group I-IV $\sigma^{70}$ proteins (and also the $\sigma^{54}$ factor) compete for a limiting pool of core RNAP complexes and target holoenzymes to cognate gene promoters. Interaction with a variety of activating and repressing transcription factors permits a complex, regulated gene expression response to changing cellular needs throughout growth and replication, during developmental processes, and in a variable, stressful environment. Finally, $\sigma$ factors are essential to the transcription initiation process and catalyze the formation of a single-stranded promoter state that is required for RNA synthesis in the cell. A rapidly burgeoning number of transcription complex structural determinations will continue to refine our understanding of transcription initiation in bacteria, and the precise molecular roles of the $\sigma$ factor in this process.

\section{Acknowledgements}

This work was supported by a grant to S.R.M. from the Natural Sciences and Engineering Research Council of Canada (Grant \# 386710). Molecular graphics were performed with the UCSF Chimera package. Chimera is developed by the Resource for Biocomputing, Visualization, and Informatics at the University of California, San Francisco (supported by NIGMS P41GM103311). 


\section{Figure Legends}

Fig. 1. Schematic representation of bacterial RNAP holoenzyme, $\sigma$ factor structure and representative promoter structure. a) subunit architecture of holoenzyme (core subunits presented in various shades of grey). $\sigma$ represented as 4 domains colour-coded according to b) regions and sub-regions of contiguous amino acid residues in $\sigma$, and indicating typical interactions with c) a representative $\sigma^{70}$ promoter with key elements indicated (EXT, extended -10 element; DISC, discriminator sequence). d) Sequence logo (Schneider and Stephens 1990) for the E. coli primary $\sigma$ factor -10 element generated from 950 sequences (Gama-Castro et al. 2015). e) Sequence logo for B. subtilis primary $\sigma$ factor -10 element generated from 656 sequences (Sierro et al. 2008).

Fig. 2. Simplified transcription scheme showing several important intermediate stages. Note that the isomerization from closed to open complex (steps 3-4) is thought to consist of at least two kinetically discernible reaction intermediates (Tsujikawa et al. 2002b) as reviewed in (Saecker et al. 2011, Ruff et al. 2015). Transcription start site (+1) indicated by angled arrow. Step 4: $\sigma$ factor melts duplex DNA and binds the non-template strand (upper line of open complex in DNA diagram) allowing template strand (lower line in DNA diagram) to enter RNAP active site (not indicated in this diagram).

Fig. 3. Interaction patterns between T. aquaticus $\sigma^{\mathrm{A}} \sigma_{2}$ amino acid residues and -10 promoter element nucleotides. a) Schematic diagram of open complex (represented in duplex DNA context) with two flipped out bases captured by domain $\sigma_{2}$. b) Re-drawing of inferred 
interactions between key amino acids ( $E$. coli $\sigma^{70}$ numbering in brackets) and the -10 element non-template strand as deduced and reported by Feklistov and Darst (2011).

Fig. 4. $\sigma$ domain $2\left(\sigma_{2}\right)$ binding pocket accommodates promoter adenine base at position -11 . a) Surface representation of T. aquaticus $\sigma^{A} \sigma_{2}$ domain and -11A nucleotide flipped out of the single-stranded stack. b) close-view indicating sub-region 2.3 amino acids participating in formation of a binding pocket that accommodates purine base (red). Thin grey line indicates local secondary structure trajectory, relevant amino acid side chain structures indicated. c) conservation of amino acids in $\sigma_{2}$ domain sub-region 2.3 amongst $T$. aquaticus, $B$. subtilis, and E. coli vegetative $\sigma$ factors. Amino acids shown in (b) highlighted in grey. Structures rendered using Chimera (Pettersen et al. 2004) from PDB file 3UGP (Feklistov and Darst 2011). 


\section{References}

Aiyar, S.E., Gourse, R.L., and Ross, W. 1998. Upstream A-tracts increase bacterial promoter activity through interactions with the RNA polymerase alpha subunit. Proc. Natl. Acad. Sci. U. S. A. 95(25): 14652-7. doi: 10.1073/pnas.95.25.14652.

Arthur, T.M., and Burgess, R.R. 1998. Localization of a sigma70 binding site on the $\mathrm{N}$ terminus of the Escherichia coli RNA polymerase beta' subunit. J. Biol. Chem. 273(47): 31381-7.

Bae, B., Feklistov, A., Lass-Napiorkowska, A., Landick, R., and Darst, S.A. 2015. Structure of a bacterial RNA polymerase holoenzyme open promoter complex. Elife 4. doi: 10.7554/eLife.08504.

Banta, A.B., Chumanov, R.S., Yuan, A.H., Lin, H., Campbell, E.A., Burgess, R.R., and Gourse, R.L. 2013. Key features of $\sigma^{S}$ required for specific recognition by $\mathrm{Crl}$, a transcription factor promoting assembly of RNA polymerase holoenzyme. Proc. Natl. Acad. Sci. U. S. A. 110(40): 15955-60. doi: 10.1073/pnas.1311642110.

Banta, A.B., Cuff, M.E., Lin, H., Myers, A.R., Ross, W., Joachimiak, A., and Gourse, R.L. 2014. Structure of the RNA polymerase assembly factor $\mathrm{Crl}$ and identification of its interaction surface with sigmaS. J. Bacteriol. 196(18): 3279-88. doi: 10.1128/JB.01910-14.

Bao, X., Nickels, B.E., and Fan, H. 2012. Chlamydia trachomatis protein GrgA activates transcription by contacting the nonconserved region of $\sigma 66$. Proc. Natl. Acad. Sci. U. S. A. 109(42):16870-5. doi: 10.1073/pnas.1207300109.

Barne, K.A., Bown, J.A., Busby, S.J.W., and Minchin, S.D. 1997. Region 2.5 of the Escherichia coli 
RNA polymerase $\sigma^{70}$ subunit is responsible for the recognition of the "extended -10 " motif at promoters. EMBO J. 16(13): 4034-4040. doi: 10.1093/emboj/16.13.4034.

Brown, N.L., Stoyanov, J. V., Kidd, S.P., and Hobman, J.L. 2003. The MerR family of transcriptional regulators. FEMS Microbiol Rev. 27(2-3):145-63. doi: 10.1016/S01686445(03)00051-2.

Browning, D.F., and Busby, S.J. 2004. The regulation of bacterial transcription initiation. Nat. Rev. Microbiol. 2(1): 57-65. doi: 10.1038/nrmicro787.

Burgess, R.R., Travers, A.A., Dunn, J.J., and Bautz, E.K. 1969. Factor stimulating transcription by RNA polymerase. Nature 221(5175): 43-6.

Campagne, S., Marsh, M.E., Capitani, G., Vorholt, J. a, and Allain, F.H.-T. 2014. Structural basis for -10 promoter element melting by environmentally induced sigma factors. Nat. Struct. Mol. Biol. 21(3): 269-76. doi: 10.1038/nsmb.2777.

Campbell, E.A., Muzzin, O., Chlenov, M., Sun, J.L., Olson, C.A., Weinman, O., Trester-Zedlitz, M.L., and Darst, S.A. 2002. Structure of the bacterial RNA polymerase promoter specificity sigma subunit. Mol. Cell 9: 527-539. doi: 10.1016/S1097-2765(02)00470-7.

Campbell, E.A., Tupy, J.L., Gruber, T.M., Wang, S., Sharp, M.M., Gross, C.A., and Darst, S.A. 2003. Crystal structure of Escherichia coli $\sigma \mathrm{E}$ with the cytoplasmic domain of its anti- $\sigma$ RseA. Mol. Cell 11(4): 1067-1078. doi: 10.1016/S1097-2765(03)00148-5.

Ciampi, M.S. 2006. Rho-dependent terminators and transcription termination. Microbiology. 152(Pt 9):2515-28. doi: 10.1099/mic.0.28982-0. 
DeHaseth, P.L., and Helmann, J.D. 1995. Open complex formation by Escherichia coli RNA polymerase: The mechanism of polymerase-induced strand separation of double helical DNA. Mol Microbiol. 16(5):817-24. doi: 10.1111/j.1365-2958.1995.tb02309.x.

Dickson, R.C., Abelson, J., Barnes, W.M., and Reznikoff, W.S. 1975. Genetic regulation: the lac control region. Science. 187(4171): 27-35.

Doolittle, R.F., Feng, D.F., Tsang, S., Cho, G., and Little, E. 1996. Determining divergence times of the major kingdoms of living organisms with a protein clock. Science 271(5248): 470-477. doi: $10.1126 /$ science.271.5248.470.

Dove, S.L., Darst, S.A., and Hochschild, A. 2003. Region 4 of sigma as a target for transcription regulation. Mol. Microbiol. 48: 863-874. doi: 3467 [pii].

Dove, S.L., Huang, F.W., and Hochschild, a. 2000. Mechanism for a transcriptional activator that works at the isomerization step. Proc. Natl. Acad. Sci. U. S. A. 97(24): 13215-13220. doi: 10.1073/pnas.97.24.13215.

Feklistov, A., and Darst, S.A. 2011. Structural basis for promoter-10 element recognition by the bacterial RNA polymerase $\sigma$ subunit. Cell 147(6): 1257-69. Elsevier Inc. doi: 10.1016/j.cell.2011.10.041.

Feklístov, A., Sharon, B.D., Darst, S. A, and Gross, C. A. 2014. Bacterial sigma factors: a historical, structural, and genomic perspective. Annu. Rev. Microbiol. 68(June): 357-76. doi: 10.1146/annurev-micro-092412-155737.

Feng, D.-F., Cho, G., and Doolittle, R.F. 1997. Determining divergence times with a protein clock: 
Update and reevaluation. Proc. Natl. Acad. Sci. 94(24): 13028-13033. doi:

10.1073/pnas.94.24.13028.

Feng, Y., Zhang, Y., and Ebright, R.H. 2016. Structural basis of transcription activation. Science 352(6291): 1330-3. doi: 10.1126/science.aaf4417.

Fenton, M.S., and Gralla, J.D. 2003. Effect of DNA bases and backbone on $\sigma 70$ holoenzyme binding and isomerization using fork junction probes. Nucleic Acids Res. 31(11): 27452750. doi: 10.1093/nar/gkg400.

Francez-Charlot, A., Frunzke, J., Reichen, C., Ebneter, J.Z., Gourion, B., and Vorholt, J.A. 2009. Sigma factor mimicry involved in regulation of general stress response. Proc Natl Acad Sci U S A 106: 3467-3472. doi: 0810291106 [pii] \r10.1073/pnas.0810291106.

Gaballa, A., MacLellan, S., and Helmann, J.D. 2012. Transcription activation by the siderophore sensor Btr is mediated by ligand-dependent stimulation of promoter clearance. Nucleic Acids Res. 40(8): 3585-3595.

Gama-Castro, S., Salgado, H., Santos-Zavaleta, A., Ledezma-Tejeida, D., Muñiz-Rascado, L., García-Sotelo, J.S., Alquicira-Hernández, K., Martínez-Flores, I., Pannier, L., CastroMondragón, J.A., Medina-Rivera, A., Solano-Lira, H., Bonavides-Martínez, C., Pérez-Rueda, E., Alquicira-Hernández, S., Porrón-Sotelo, L., López-Fuentes, A., Hernández-Koutoucheva, A., Moral-Chávez, V. Del, Rinaldi, F., and Collado-Vides, J. 2015. RegulonDB version 9.0: high-level integration of gene regulation, coexpression, motif clustering and beyond. Nucleic Acids Res. 44(D1): gkv1156. doi: 10.1093/nar/gkv1156. 
Gardella, T., Moyle, H., and Susskind, M.M. 1989. A mutant Escherichia coli sigma 70 subunit of RNA polymerase with altered promoter specificity. J. Mol. Biol. 206(4): 579-90.

Geszvain, K., Gruber, T.M., Mooney, R.A., Gross, C.A., and Landick, R. 2004. A hydrophobic patch on the flap-tip helix of E.coli RNA polymerase mediates sigma(70) region 4 function. J. Mol. Biol. 343(3): 569-87. doi: 10.1016/j.jmb.2004.08.063.

Goldman, S.R., Ebright, R.H., and Nickels, B.E. 2009. Direct detection of abortive RNA transcripts in vivo. Science 324(5929): 927-8. doi: 10.1126/science.1169237.

Gralla, J.D., Carpousis, A.J., and Stefano, J.E. 1980. Productive and abortive initiation of transcription in vitro at the lac UV5 promoter. Biochemistry 19(25): 5864-5869. doi: 10.1021/bi00566a031.

Gribskov, M., and Burgess, R.R. 1986. Sigma factors from E. coli, B. subtilis, phage SP01, and phage T4 are homologous proteins. Nucleic Acids Res. 14(16): 6745-6763. doi: 10.1093/nar/14.16.6745.

Gruber, T.M., and Gross, C.A. 2003. Multiple sigma subunits and the partitioning of bacterial transcription space. Annu. Rev. Microbiol. 57: 441-466. doi: 10.1146/annurev.micro.57.030502.090913.

Gusarov, I., and Nudler, E. 1999. The mechanism of intrinsic transcription termination. Mol. Cell 3(4): 495-504. doi: 10.1016/S1097-2765(00)80477-3.

Haugen, S.P., Berkmen, M.B., Ross, W., Gaal, T., Ward, C., and Gourse, R.L. 2006. rRNA Promoter Regulation by Nonoptimal Binding of $\sigma$ Region 1.2: An Additional Recognition 
Element for RNA Polymerase. Cell 125(6): 1069-1082. doi: 10.1016/j.cell.2006.04.034.

Heinrich, J., and Wiegert, T. 2009. Regulated intramembrane proteolysis in the control of extracytoplasmic function sigma factors. Res. Microbiol. 160(9): 696-703. doi: 10.1016/j.resmic.2009.08.019.

Heldwein, E.E., and Brennan, R.G. 2001. Crystal structure of the transcription activator BmrR bound to DNA and a drug. Nature 409(6818): 378-382. doi: 10.1038/35053138.

Helmann, J.D. 1999. Anti-sigma factors. Curr Opin Microbiol. 2(2): 135-141. doi: 10.1016/S1369$5274(99) 80024-1$.

Helmann, J.D., and Chamberlin, M.J. 1988. Structure and function of bacterial sigma factors. Annu. Rev. Biochem. 57: 839-72. doi: 10.1146/annurev.bi.57.070188.004203.

Hendershot, J.M., and O'Brien, P.J. 2014. Critical role of DNA intercalation in enzyme-catalyzed nucleotide flipping. Nucleic Acids Res. 42(20): 12681-90. doi: 10.1093/nar/gku919.

Hook-Barnard, I.G., and Hinton, D.M. 2007. Transcription initiation by mix and match elements: flexibility for polymerase binding to bacterial promoters. Gene Regul. Syst. Biol. 1(301): 275-293.

Hubin, E.A., Tabib-Salazar, A., Humphrey, L.J., Flack, J.E., Olinares, P.D.B., Darst, S.A., Campbell, E.A., and Paget, M.S. 2015. Structural, functional, and genetic analyses of the actinobacterial transcription factor RbpA. Proc. Natl. Acad. Sci. U. S. A. 112(23): 71717176. doi: 10.1073/pnas.1504942112. 
Juang, Y.L., and Helmann, J.D. 1994. A promoter melting region in the primary sigma factor of Bacillus subtilis. Identification of functionally important aromatic amino acids. J. Mol. Biol. 235: 1470-1488. doi: S0022-2836(84)71102-8 [pii] \n10.1006/jmbi.1994.1102 [doi].

Kang, J.G., Paget, M.S.B., Seok, Y.J., Hahn, M.Y., Bae, J.B., Hahn, J.S., Kleanthous, C., Buttner, M.J., and Roe, J.H. 1999. RsrA, an anti-sigma factor regulated by redox change. EMBO J. 18: 4292-4298. doi: 10.1093/emboj/18.15.4292.

Karpen, M.E., and deHaseth, P.L. 2015. Base flipping in open complex formation at bacterial promoters. Biomolecules 5(2): 668-78. doi: 10.3390/biom5020668.

Keilty, S., and Rosenberg, M. 1987. Constitutive function of a positively regulated promoter reveals new sequences essential for activity. J. Biol. Chem. 262(13): 6389-6395.

Kenney, T.J., and Moran, C.P.J. 1991. Genetic evidence for interaction of sigma A with two promoters in Bacillus subtilis. J. Bacteriol. 173(11): 3282-3290.

Lane, W.J., and Darst, S.A. 2006. The structural basis for promoter -35 element recognition by the group IV $\sigma$ factors. PLoS Biol. 4: 1491-1500. doi: 10.1371/journal.pbio.0040269.

Lee, D.J., Minchin, S.D., and Busby, S.J.W. 2012. Activating transcription in bacteria. Annu. Rev. Microbiol. 66: 125-52. doi: 10.1146/annurev-micro-092611-150012.

Lim, H.M., Lee, H.J., Roy, S., and Adhya, S. 2001. A "master" in base unpairing during isomerization of a promoter upon RNA polymerase binding. Proc Natl Acad Sci U S A 98(26): 14849-14852. doi: 10.1073/pnas.261517398. 
Liu, B., Zuo, Y., and Steitz, T.A. 2016. Structures of E. coli $\sigma$ S-transcription initiation complexes provide new insights into polymerase mechanism. Proc. Natl. Acad. Sci. U. S. A. 113(15): 4051-4056. doi: 10.1073/pnas.1520555113.

Lonetto, M.A., Rhodius, V., Lamberg, K., Kiley, P., Busby, S., and Gross, C. 1998. Identification of a contact site for different transcription activators in region 4 of the Escherichia coli RNA polymerase $\sigma 70$ subunit. J. Mol. Biol. 284(5): 1353-1365. doi: 10.1006/jmbi.1998.2268.

Lonetto, M., Gribskov, M., and Gross, C.A. 1992. The sigma 70 family: sequence conservation and evolutionary relationships. J. Bacteriol. 174(12): 3843-9.

MacLellan, S.R., Guariglia-Oropeza, V., Gaballa, A., and Helmann, J.D. 2009. A two-subunit bacterial sigma-factor activates transcription in Bacillus subtilis. Proc. Natl. Acad. Sci. U. S. A. 106: 21323-21328. doi: 10.1073/pnas.0910006106.

MacLellan, S.R., Wecke, T., and Helmann, J.D. 2008. A previously unidentified $\sigma$ factor and two accessory proteins regulate oxalate decarboxylase expression in Bacillus subtilis. Mol. Microbiol. 69: 954-967. doi: 10.1111/j.1365-2958.2008.06331.x.

Malhotra, A., Severinova, E., and Darst, S.A. 1996. Crystal structure of a sigma 70 subunit fragment from E. coli RNA polymerase. Cell 87: 127-136. doi: 10.1016/S00928674(00)81329-X.

Mekler, V., and Severinov, K. 2013. Cooperativity and interaction energy threshold effects in recognition of the -10 promoter element by bacterial RNA polymerase. Nucleic Acids Res. 41(15): 7276-7285. doi: 10.1093/nar/gkt541. 
Missiakas, D., and Raina, S. 1998. The extracytoplasmic function sigma factors: role and regulation. Mol. Microbiol. 28(6): 1059-66.

Murakami, K. 2015. Structural Biology of Bacterial RNA Polymerase. Biomolecules 5(2): 848864. doi: 10.3390/biom5020848.

Murakami, K.S., and Darst, S.A. 2003. Bacterial RNA polymerases : the whole story. Curr. Opin. Struct. Biol. 13: 31-39. doi: 10.1016/S0959-440X(02)00005-2.

Murakami, K.S., Masuda, S., and Darst, S.A. 2002. Structural basis of transcription initiation: RNA polymerase holoenzyme at 4 A resolution. Science 296: 1280-1284. doi: 10.1126/science.1069594.

Niu, W., Kim, Y., Tau, G., Heyduk, T., and Ebright, R.H. 1996. Transcription activation at class II CAP-dependent promoters: Two interactions between CAP and RNA polymerase. Cell 87(6): 1123-1134. doi: 10.1016/S0092-8674(00)81806-1.

Paget, M.S. 2015. Bacterial Sigma Factors and Anti-Sigma Factors: Structure, Function and Distribution. Biomolecules 5(3): 1245-65. doi: 10.3390/biom5031245.

Paget, M.S.B., and Helmann, J.D. 2003. The sigma70 family of sigma factors. Genome Biol. 4: 203. doi: 10.1186/gb-2003-4-1-203.

Paget, M.S.B., Molle, V., Cohen, G., Aharonowitz, Y., and Buttner, M.J. 2001. Defining the disulphide stress response in Streptomyces coelicolor A3(2): Identification of the sigmaR regulon. Mol. Microbiol. 42(4): 1007-1020. doi: 10.1046/j.1365-2958.2001.02675.x. 
Panaghie, G., Aiyar, S.E., Bobb, K.L., Hayward, R.S., and de Haseth, P.L. 2000. Aromatic amino acids in region 2.3 of Escherichia coli sigma 70 participate collectively in the formation of an RNA polymerase-promoter open complex. J. Mol. Biol. 299(5): 1217-30. doi: 10.1006/jmbi.2000.3808.

Pané-Farré, J., Lewis, R.J., and Stülke, J. 2005. The RsbRST stress module in bacteria: A signalling system that may interact with different output modules. J Mol Microbiol Biotechnol. 9(2):65-76. doi: 10.1159/000088837.

Pettersen, E.F., Goddard, T.D., Huang, C.C., Couch, G.S., Greenblatt, D.M., Meng, E.C., and Ferrin, T.E. 2004. UCSF Chimera--a visualization system for exploratory research and analysis. J. Comput. Chem. 25(13): 1605-12. doi: 10.1002/jcc.20084.

Pratt, L.A., and Silhavy, T.J. 1998. Crl stimulates RpoS activity during stationary phase. Mol. Microbiol. 29(5): 1225-1236. doi: 10.1046/j.1365-2958.1998.01007.x.

Qiu, J., and Helmann, J.D. 1999. Adenines at $-11,-9$ and -8 play a key role in the binding of Bacillus subtilis Esigma(A) RNA polymerase to -10 region single-stranded DNA. Nucleic Acids Res. 27: 4541-4546. doi: gkc673 [pii].

Roberts, R.J., and Cheng, X. 1998. Base flipping. Annu. Rev. Biochem. 67: 181-98. doi: 10.1146/annurev.biochem.67.1.181.

Rong, J.C., and Helmann, J.D. 1994. Genetic and physiological studies of Bacillus subtilis sigma A mutants defective in promoter melting. J. Bacteriol. 176(17): 5218-24.

Ruff, E.F., Record, M.T., and Artsimovitch, I. 2015. Initial events in bacterial transcription 
initiation. Biomolecules 5(2): 1035-62. doi: 10.3390/biom5021035.

Saecker, R.M., Record, M.T., and Dehaseth, P.L. 2011. Mechanism of bacterial transcription initiation: RNA polymerase - Promoter binding, isomerization to initiation-competent open complexes, and initiation of RNA synthesis. J Mol Biol. 412(5):754-71. doi: 10.1016/j.jmb.2011.01.018.

Sanderson, A., Mitchell, J.E., Minchin, S.D., and Busby, S.J.W. 2003. Substitutions in the Escherichia coli RNA polymerase $\sigma 70$ factor that affect recognition of extended -10 elements at promoters. FEBS Lett. 544(1-3): 199-205. doi: 10.1016/S0014-5793(03)005003.

Schneider, T.D., and Stephens, R.M. 1990. Sequence logos: a new way to display consensus sequences. Nucleic Acids Res. 18(20): 6097-6100. doi: 10.1093/nar/18.20.6097.

Siegele, D.A., Hu, J.C., Walter, W.A., and Gross, C.A. 1989. Altered promoter recognition by mutant forms of the sigma 70 subunit of Escherichia coli RNA polymerase. J. Mol. Biol. 206(4): 591-603.

Sierro, N., Makita, Y., De hoon, M., and Nakai, K. 2008. DBTBS: A database of transcriptional regulation in Bacillus subtilis containing upstream intergenic conservation information. Nucleic Acids Res. 36(SUPPL. 1). doi: 10.1093/nar/gkm910.

Staroń, A., Sofia, H.J., Dietrich, S., Ulrich, L.E., Liesegang, H., and Mascher, T. 2009. The third pillar of bacterial signal transduction: Classification of the extracytoplasmic function (ECF) $\sigma$ factor protein family. Mol. Microbiol. 74(3): 557-581. doi: 10.1111/j.1365- 
2958.2009.06870.x.

Tabib-Salazar, A., Liu, B., Doughty, P., Lewis, R.A., Ghosh, S., Parsy, M.L., Simpson, P.J., O’Dwyer, K., Matthews, S.J., and Paget, M.S. 2013. The actinobacterial transcription factor RbpA binds to the principal sigma subunit of RNA polymerase. Nucleic Acids Res. 41(11): 56795691. doi: 10.1093/nar/gkt277.

Travers, A.A. 1980. Promoter sequence for stringent control of bacterial ribonucleic acid synthesis. J. Bacteriol. 141(2): 973-976.

Tsujikawa, L., Strainic, M.G., Watrob, H., Barkley, M.D., and DeHaseth, P.L. 2002a. RNA polymerase alters the mobility of an A-residue crucial to polymerase-induced melting of promoter DNA. Biochemistry 41(51): 15334-15341. doi: 10.1021/bi026539m.

Tsujikawa, L., Tsodikov, O. V, and deHaseth, P.L. 2002b. Interaction of RNA polymerase with forked DNA: evidence for two kinetically significant intermediates on the pathway to the final complex. Proc. Natl. Acad. Sci. U. S. A. 99(6): 3493-8. National Academy of Sciences. doi: 10.1073/pnas.062487299.

Vassylyev, D.G., Sekine, S., Laptenko, O., Lee, J., Vassylyeva, M.N., Borukhov, S., and Yokoyama, S. 2002. Crystal structure of a bacterial RNA polymerase holoenzyme at 2.6 A resolution. Nature 417(6890): 712-719. doi: 10.1038/nature752.

Xue, X., Davis, M.C., Steeves, T., Bishop, A., Breen, J., MacEacheron, A., Kesthely, C.A., Hsu, F., and MacLellan, S.R. 2016. Characterization of a protein-protein interaction within the SigORsoA two-subunit factor: the 70 region 2.3-like segment of RsoA mediates interaction with 
SigO. Microbiology. In press. doi: 10.1099/mic.0.000358.

Zhang, N., and Buck, M. 2015. A perspective on the enhancer dependent bacterial RNA polymerase. Biomolecules 5(2): 1012-9. doi: 10.3390/biom5021012.

Zhang, Y., Feng, Y., Chatterjee, S., Tuske, S., Ho, M.X., Arnold, E., and Ebright, R.H. 2012. Structural basis of transcription initiation. Science 338(6110): 1076-80. doi: 10.1126/science.1227786.

Zuber, P., Healy, J., Carter, H.L., Cutting, S., Moran, C.P., and Losick, R. 1989. Mutation changing the specificity of an RNA polymerase sigma factor. J. Mol. Biol. 206(4): 605-14.

Zuo, Y., and Steitz, T.A. 2015. Crystal Structures of the E. coli Transcription Initiation Complexes with a Complete Bubble. Mol. Cell 58(3): 534-540. doi: 10.1016/j.molcel.2015.03.010.

Figure Legends 
Fig. 1. Schematic representation of bacterial RNAP holoenzyme, $\sigma$ factor structure and representative promoter structure. a) subunit architecture of holoenzyme (core subunits presented in various shades of grey). $\sigma$ represented as 4 domains colour-coded according to b) regions and sub-regions of contiguous amino acid residues in $\sigma$, and indicating typical interactions with c) a representative $\sigma^{70}$ promoter with key elements indicated (EXT, extended -10 element; DISC, discriminator sequence). d) Sequence logo (Schneider and Stephens 1990) for the $E$. coli primary $\sigma$ factor -10 element generated from 950 sequences (Gama-Castro et al. 2015). e) Sequence logo for B. subtilis primary $\sigma$ factor -10 element generated from 656 sequences (Sierro et al. 2008).

Fig. 2. Simplified transcription scheme showing several important intermediate stages. Note that the isomerization from closed to open complex (steps 3-4) is thought to consist of at least two kinetically discernible reaction intermediates (Tsujikawa et al. 2002b) as reviewed in (Saecker et al. 2011, Ruff et al. 2015). Transcription start site (+1) indicated by angled arrow. Step 4: $\sigma$ factor melts duplex DNA and binds the non-template strand (upper line of open complex in DNA diagram) allowing template strand (lower line in DNA diagram) to enter RNAP active site (not indicated in this diagram).

Fig. 3. Interaction patterns between T. aquaticus $\sigma^{\mathrm{A}} \sigma_{2}$ amino acid residues and -10 promoter element nucleotides. a) Schematic diagram of open complex (represented in duplex DNA context) with two flipped out bases captured by domain $\sigma_{2}$. b) Re-drawing of inferred interactions between key amino acids ( $E$. coli $\sigma^{70}$ numbering in brackets) and the -10 element non-template strand as deduced and reported by Feklistov and Darst (2011). 
Fig. 4. $\sigma$ domain $2\left(\sigma_{2}\right)$ binding pocket accommodates promoter adenine base at position -11. a) Surface representation of T. aquaticus $\sigma^{\mathrm{A}} \sigma_{2}$ domain and -11A nucleotide flipped out of the single-stranded stack. b) close-view indicating sub-region 2.3 amino acids participating in formation of a binding pocket that accommodates purine base (red). Thin grey line indicates local secondary structure trajectory, relevant amino acid side chain structures indicated. c) conservation of amino acids in $\sigma_{2}$ domain sub-region 2.3 amongst $T$. aquaticus, $B$. subtilis, and E. coli vegetative $\sigma$ factors. Amino acids shown in (b) highlighted in grey. Structures rendered using Chimera (Pettersen et al. 2004) from PDB file 3UGP (Feklistov and Darst 2011). 


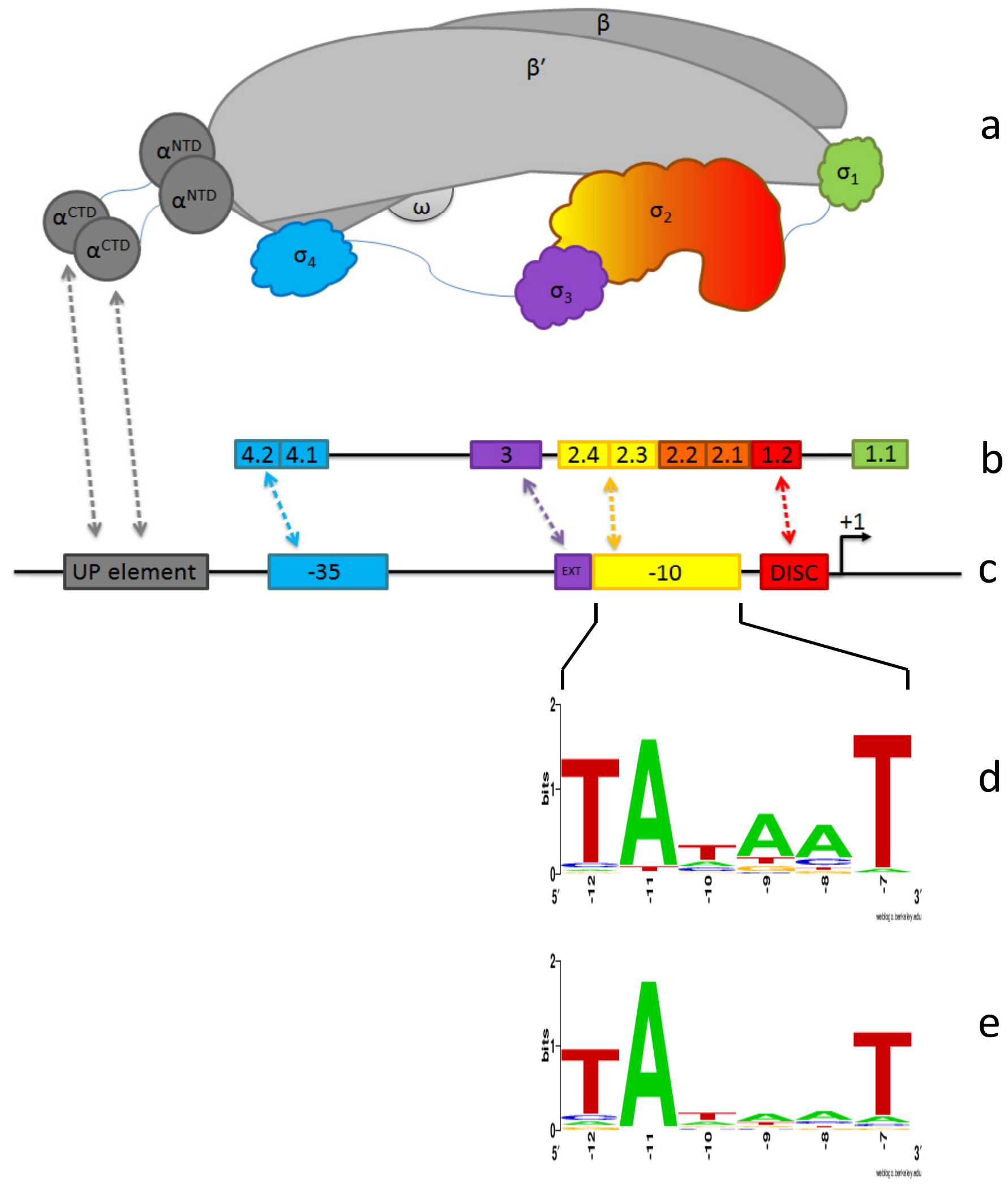


1

\section{core}

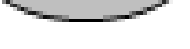

2

3

\section{holo}

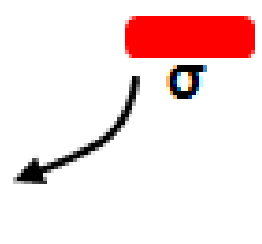

4

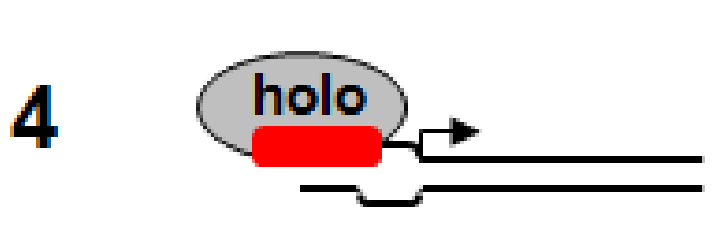

holo
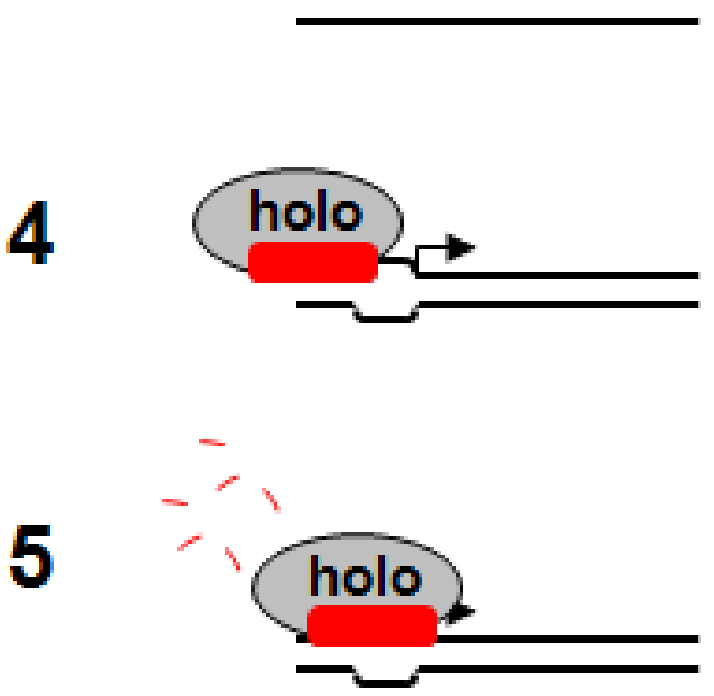

6

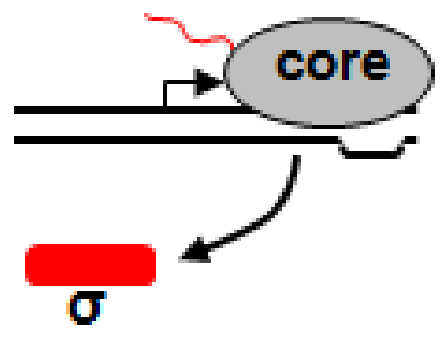

RNAP core assembly $\alpha_{2} \beta \beta$ ' $\omega$ subunits

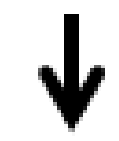

RNAP holoenzyme formation $\alpha_{2} \beta \beta$ ' $\omega \sigma$

DNA scanning promoter recognition and binding

Pre-initiation closed complex

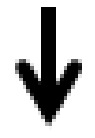

Pre-initiation open complex

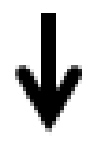

Abortive transcription

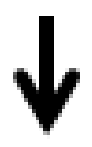

Promoter escape $\sigma$ factor release

Elongation

Termination 
a

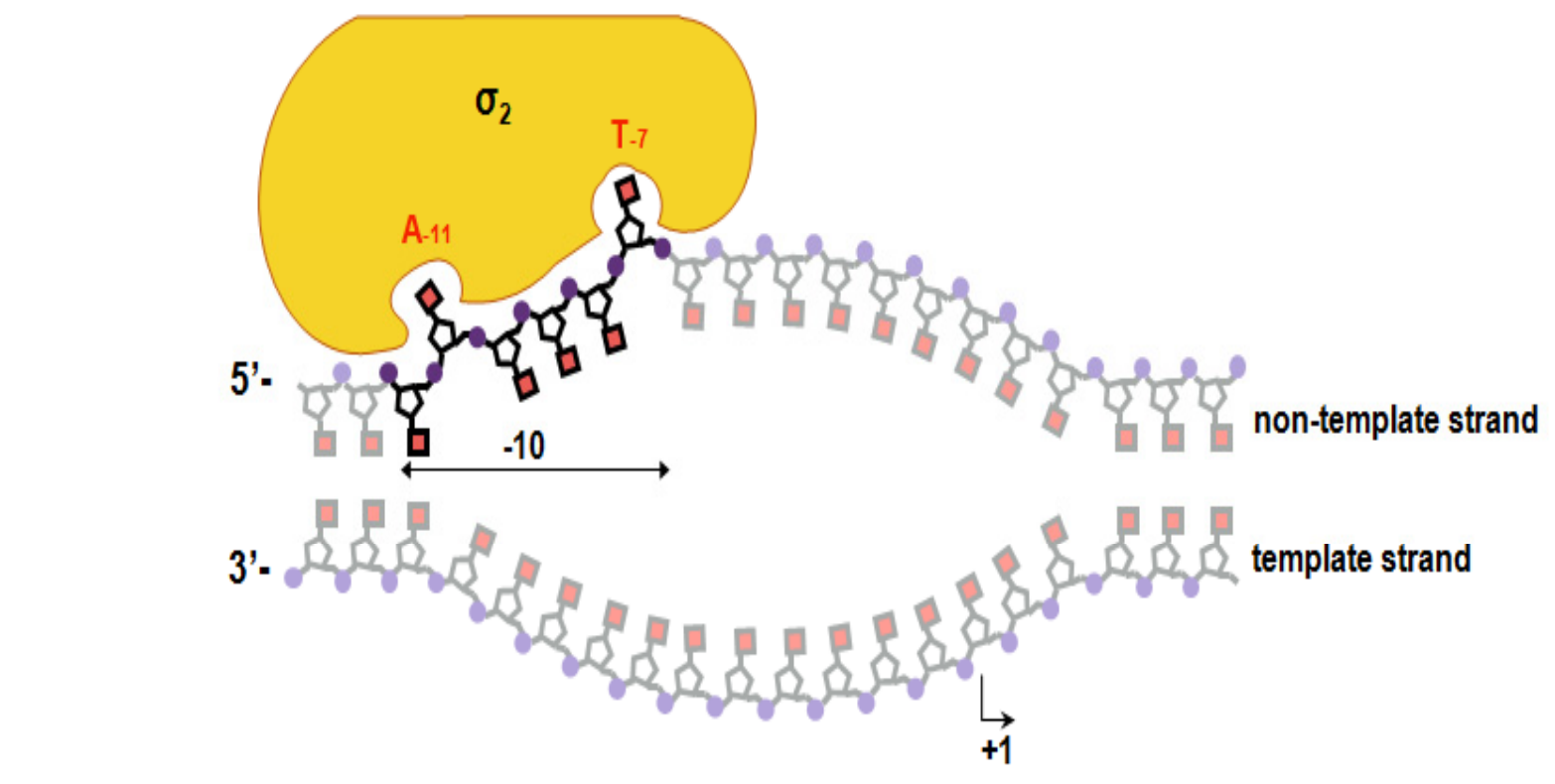

b

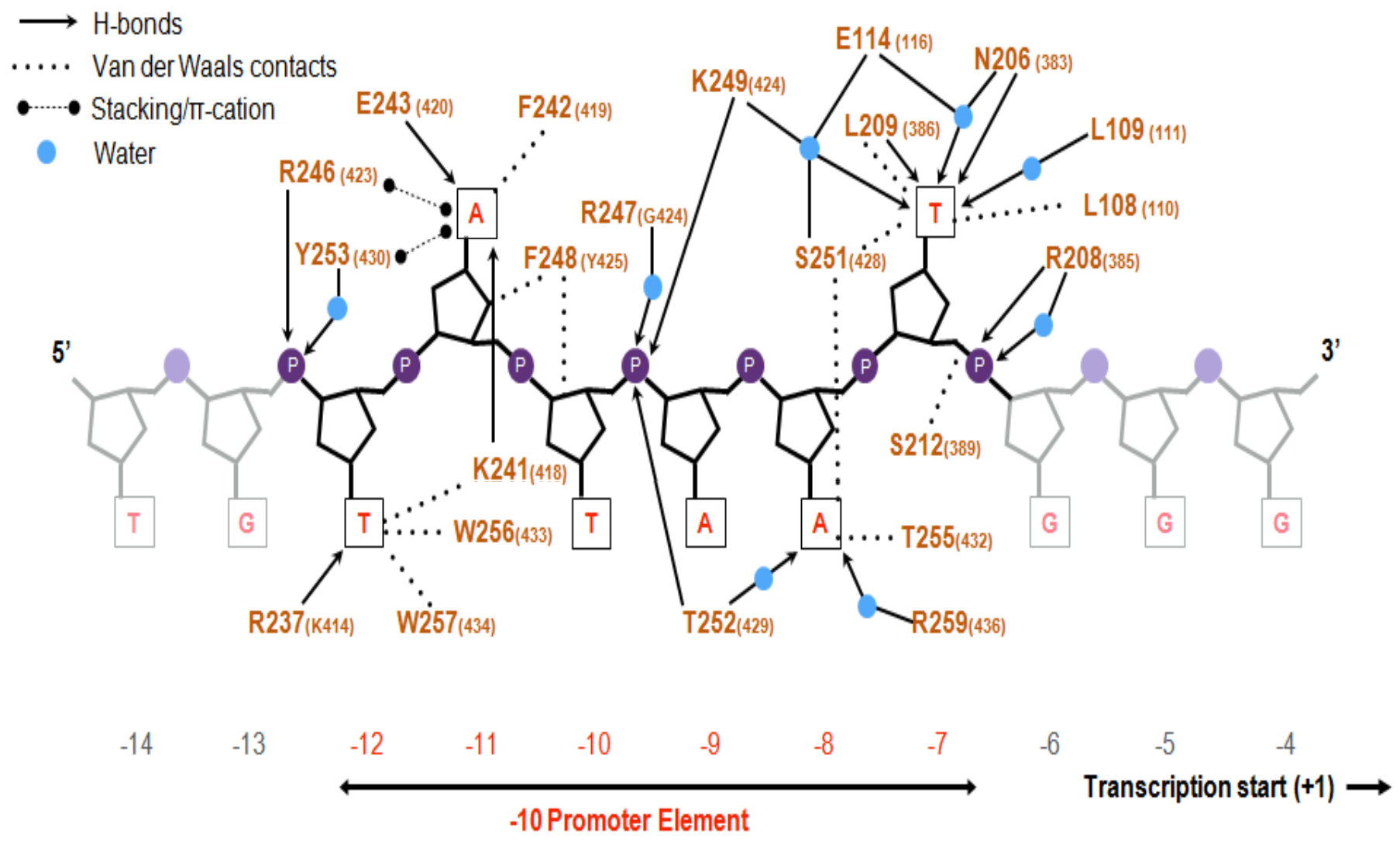




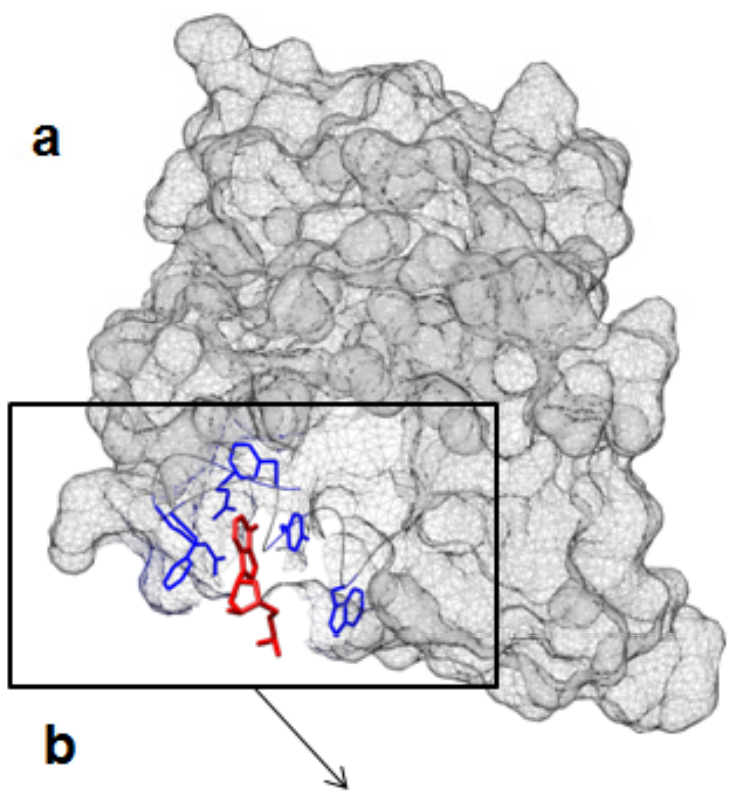

C

$\begin{array}{ll}\text { Taq } \sigma^{\mathrm{A}} & 238 \text {-AVEKFEYKRRFKFSTYATWW } \\ \text { Bsu } \sigma^{\mathrm{A}} & 174 \text {-AVEKFDYRKGYKFSTYATWW } \\ \text { Eco } \sigma^{70} & 415 \text {-AVDKFEYRRGYKFSTYATWW }\end{array}$

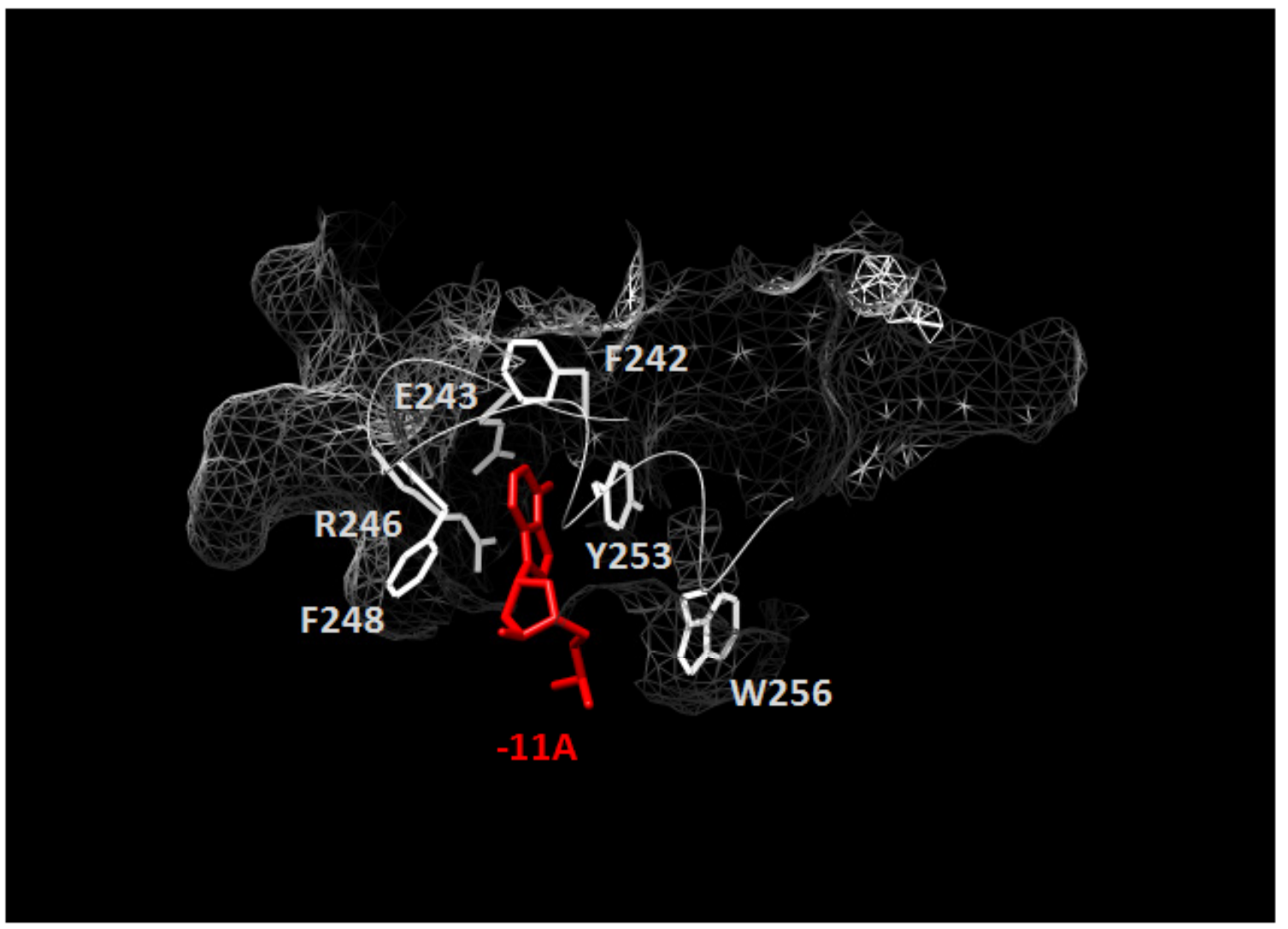

